\title{
ON THE SEMIPRIMITIVITY OF SKEW POLYNOMIAL RINGS
}

\author{
by A. MOUSSAVI
}

(Received 24th January 1989, revised 20th April 1992)

\begin{abstract}
Let $R$ be a left Noetherian ring with the ascending chain condition on right annihilators, let $\alpha$ be a ring monomorphism of $R$ and $\delta$ an $\alpha$-derivation of $R$. We prove that, if $R$ is semiprime or $\alpha$-prime, then $R[X ; \alpha, \delta]$ is semiprimitive (and left Goldie), and that $J(R[X ; \alpha])$ equals $N(R)[X ; \alpha]$.
\end{abstract}

1991 Mathematics subject classification: 16S36, 16N20

Let $R$ be a ring. A well known result of Amitsur [1] states that if $R$ has no nil ideals then the polynomial ring $R[x]$ is semiprimitive. Various authors, for example Bedi and Ram [2], Bell [3], and C. R. Jordan and D. A. Jordan [13], have extended this result to skew polynomial rings of the form $R[x ; \alpha, \delta]$, where $\alpha$ is an automorphism of $R$, and $\delta$ is an $\alpha$-derivation of $R$. Most of these have worked either with the case $\delta=0$ and $\alpha$ an automorphism or the case where $\alpha$ is the identity. El Ahmar [8] has shown that, if $R$ is right and left Noetherian, $\alpha: R \rightarrow R$ a monomorphism, then $R[x ; \alpha]$ is semiprimitive. An example $[13, \S 5]$ shows that some conditions on $R$ and $\alpha$ are necessary if results of this kind are to be valid. Bell [3] has shown that if $R$ is semiprime left Goldie with $\alpha: R \rightarrow R$ an automorphism and $\delta$ an $\alpha$-derivation then $R[x ; \alpha, \delta]$ is semiprimitive left Goldie, and has commented that it is not known whether this generalizes to the case where $\alpha$ is not surjective.

The situation we shall be concerned with is that of a ring $R$ and a monomorphism $\alpha$ : $R \rightarrow R$ which is not assumed to be surjective. Let $R$ be a left Noetherian ring with ascending chain condition on right annihilators, $N(R)$ its nilpotent radical. Dean [7] has shown that $\alpha(N(R)) \subseteq N(R)$. We use methods adapted from those of some of the above authors together with Dean's result and the construction of the ring $A(R, \alpha)$ of Jordan [14], to show that $R[x ; \alpha, \delta]$ is semiprimitive left Goldie if $R$ is semiprime or $\alpha$-prime. We also show that if $R$ is semiprime left or right Goldie and $\alpha: R \rightarrow R$ is a monomorphism then $R[x ; \alpha]$ is semiprimitive. We use this result to see that if $R$ is left Noetherian with ascending chain condition on right annihilators then

$$
J(R[x ; \alpha])=N(R[x ; \alpha])=N(R)[x ; \alpha]
$$

\section{Preliminaries}

All rings in this paper have 1 and all endomorphisms are assumed to preserve 1. 
Let $R$ be a ring and $\alpha: R \rightarrow R$ a monomorphism. An $\alpha$-derivation is an additive map $\delta$ from $R$ to $R$ such that $\delta(a b)=\delta(a) b+\alpha(a) \delta(b)$ for all $a, b \in R$. Given such an $\alpha$ and $\delta$, the Ore extension $R[x ; \alpha, \delta]$ is the ring of all formal linear combinations $\sum_{i=0}^{n} r_{i} x^{i}, r_{i} \in R$, $n \geqq 0$, with multiplication subject to the relation $x r=\alpha(r) x+\delta(r)$. If $\delta=0$ we have the skew polynomial ring $R[x ; \alpha]$. Then the set $\left\{x^{i}\right\}_{i \geq 1}$ is easily seen to be a left Ore set so that one can localize and form the skew Laurent polynomial ring, $R\left[x, x^{-1} ; \alpha\right]$. Elements of $R\left[x, x^{-1} ; \alpha\right]$ are finite sums of elements of the form $x^{-i} r x^{j}$ where $r \in R, i \geqq 0$, $j \geqq 0$. Multiplication is subject to $r x^{-1}=x^{-1} \alpha(r)$ for all $r \in R$.

In [14], D. A. Jordan has constructed an overring $A(R, \alpha)$ of $R$, which is, in a sense, the minimal overring of $R$ to which $\alpha$ extends as an automorphism. Consider an element of $R\left[x, x^{-1} ; \alpha\right]$ of the form $x^{-i} r x^{i}, r \in R, i \geqq 0$. Then, for $j \geqq 0, x^{-j} r x^{j}=x^{-(i+j)} \alpha^{i}(r) x^{(i+j)}$. It follows that the set of all such elements forms a subring of $R\left[x, x^{-1} ; \alpha\right]$, with

$$
\begin{gathered}
\left.x^{-i} r x^{i}+x^{-j} s x^{j}=x^{-(i+j)}\left(\alpha^{j}(r)+\alpha^{i}(s)\right) x^{(i+j}\right) \text { and } \\
z^{-i} r x^{i} \cdot x^{-j} s x^{j}=x^{-(i+j)}\left(\alpha^{j}(r) \cdot \alpha^{i}(s)\right) x^{(i+j)}, \text { with } r, s \in R .
\end{gathered}
$$

This subring is denoted $A(R, \alpha)$. We extend $\alpha$ to $A(R, \alpha)$, by setting $\alpha\left(x^{-i} r x^{i}\right)=x^{-i} \alpha(r) x^{i}$. Since $\alpha\left(x^{-(i+1)} r x^{(i+1)}\right)=x^{-i} r x^{i}, \alpha$ is an automorphism of $A(R, \alpha)$.

Definition 1.1. Let $I$ be an ideal of a ring $R, \alpha: R \rightarrow R$ be a monomorphism. Then $I$ is said to be an $\alpha$-ideal if $\alpha(I) \subseteq I$; I is said to be $\alpha$-invariant if $\alpha^{-1}(I)=I ; I$ is said to be $\alpha$-prime if it is $\alpha$-invariant and for any $\alpha$-ideals $A$ and $B$ of $R, A B \subseteq I$ implies that $A \subseteq I$ or $B \subseteq I$. The ring $R$ is $\alpha$-prime if 0 is an $\alpha$-prime ideal of $R$.

Lemma 1.2. The ring $R$ is $\alpha$-prime if and only if, for $a, b \in R, \alpha^{t}(a) R \alpha^{s}(b)=0$ for all $t \geqq 0, s \geqq 0$, implies that $a=0$ or $b=0$.

Proof. The proof is straightforward.

Definition 1.3. We mean by a right annihilator ideal (left annihilator ideal), an ideal of the form rann $I$, (respectively lann $I$ ), where $I$ is an ideal of $R$. If an ideal of $R$ is of the form $\operatorname{rann} I=$ lann $I$, where $I$ is an $\alpha$-ideal of $R$, then it is said to be an annihilator $\alpha$-ideal.

By an annihilator $\alpha$-prime ideal, we mean an annihilator $\alpha$-ideal which is also $\alpha$-prime.

The nilpotent radical of a ring $R$ will be taken to be the sum of the nilpotent ideals of $R$ and will be denoted by $N(R)$. The Jacobson radical of $R$ will be denoted by $J(R)$.

Lemma 1.4. Let $R$ be a ring satisfying the ascending chain condition on right annihilators and let I be an $\alpha$-ideal of $R$. Then rann I is an $\alpha$-invariant ideal.

Proof. Since rann $I \subseteq \operatorname{rann}(\alpha(I)) \subseteq \operatorname{rann}\left(\alpha^{2}(I)\right) \subseteq \ldots$, we have that, for some $k \geqq 0$, $\operatorname{rann}\left(\alpha^{k}(I)\right)=\operatorname{rann}\left(\alpha^{k+1}(I)\right)$. Let $a \in \operatorname{rann} I$. Then $\alpha^{k+1}(a) \in \operatorname{rann}\left(\alpha^{k}(I)\right)$, so $\alpha(a) \in \operatorname{rann} I$. If $\alpha(a) \in \operatorname{rann} I$, then $\alpha^{k+1}(a) \in \operatorname{rann}\left(\alpha^{k+1}(I)\right)$, so $a \in \operatorname{rann} I$. 
Lemma 1.5. Let $R$ be a ring satisfying the ascending chain condition on right annihilators and $P$ be an $\alpha$-invariant ideal of $R$. Then, (i) for each $t \geqq 1, P \subseteq$ $\operatorname{lann}\left(\alpha^{t}(\operatorname{rann}(P))\right)$, and, (ii) if $P=\operatorname{lann} L$ for a subset $L$ of $R$, then lann $L \subseteq \operatorname{lann}\left(\alpha^{t}(L)\right)$, $t \geqq 1$.

Proof. (i) The chain rann $P \subseteq \operatorname{rann}(\alpha(P)) \subseteq \ldots$ terminates. So for some integer $k \geqq 0$ and for all $t \geqq 0, \operatorname{rann}\left(\alpha^{k}(P)\right)=\operatorname{rann}\left(\alpha^{k+t}(P)\right)$. Let $t \geqq 1$, and $b \in \operatorname{rann} P$. Then $P b=0$, so $\alpha^{k+t}(b) \in \operatorname{rann}\left(\alpha^{k}(P)\right)$. Since $\alpha^{k}$ is injective $P \alpha^{t}(b)=0$.

(ii) We have for each $t \geqq 1, P=\operatorname{lann} L \subseteq \operatorname{lann}\left(\alpha^{t}(\operatorname{rann}(P))\right)=\operatorname{lann}\left(\alpha^{t}(\operatorname{rann}(\operatorname{lann}(L)))\right)$. But $\alpha^{t}(L) \subseteq \alpha^{t}(\operatorname{rann}(\operatorname{lann}(L)))$. Thus

$$
\operatorname{lann}\left(\alpha^{t}(\operatorname{rann}(\operatorname{lann}(L)))\right) \subseteq \operatorname{lann}\left(\alpha^{t}(L)\right), t \geqq 1
$$

Proposition 1.6. Let $R$ be a semiprime ring satisfying the ascending chain condition on right annihilators. Then $R$ has only a finite number of minimal $\alpha$-prime ideals, and their intersection is zero. An $\alpha$-prime ideal of $R$ is minimal if and only if it is an annihilator $\alpha$-ideal.

Proof. First we show that each annihilator $\alpha$-ideal of $R$ contains a product of annihilator $\alpha$-prime ideals. Suppose not and let $L$ be an annihilator $\alpha$-ideal of $R$ which is maximal with respect to not containing a product of annihilator $\alpha$-prime ideals. So $L=\operatorname{rann} I=$ lann $I$, for some $\alpha$-ideal $I$ of $R$. Now, $L$ cannot be an $\alpha$-prime ideal, otherwise it is, itself, an annihilator $\alpha$-prime ideal. Hence there are $\alpha$-ideals $T$ and $K$ of $R$ which strictly contain $L$ such that $T K \subseteq L$. Take $C=\operatorname{rann}(I T)$ and $B=\operatorname{lann}(C T)$. By [16, Proposition 2.2.14], $C=\operatorname{rann}(I T)=\operatorname{lann}(I T)$, and $B=\operatorname{lann}(C I)=\operatorname{rann}(C I)$. Since $I L=0$ and $T K \subseteq L, I T K=0$. Since $I T \subseteq I, L=\operatorname{rann} I \subseteq \operatorname{rann}(I T)$, whence $C \supseteq L$. Also $B=\operatorname{lann}(C I) \supseteq \operatorname{lann} I$, so $B \supseteq L$. But $B=\operatorname{lann}(C I)$ thus $B C \subseteq \operatorname{lann} I=L$. Since $I$ and $T$ are $\alpha$-ideals, $C$ is an annihilator $\alpha$-ideal. Also $B$ is an annihilator $\alpha$-ideal. We have $I T K=0$, and $C I T=0$, so $K \subseteq C$ and $T \subseteq B$. Since $T$ and $K$ strictly contain $L$, the annihilator $\alpha$-ideals $B$ and $C$ strictly contain $L$ with $B C \subseteq L$. By the choice of $L, B$ and $C$ each contain a product of annihilator $\alpha$-prime ideals so also does $L$. Therefore we can deduce that every annihilator $\alpha$-ideal of $R$ contains a product of annihilator $\alpha$-prime ideals. Since the zero ideal of $R$ is an annihilator $\alpha$-ideal, there are annihilator $\alpha$-prime ideals $P_{1}, P_{2}, \ldots, P_{n}$ of $R$ say, such that $P_{1} P_{2} \ldots P_{n}=0$. We have $\left(P_{1} \cap P_{2} \cap \cdots \cap P_{n}\right)^{n}=0$. Since $R$ is semiprime, $P_{1} \cap P_{2} \cap \cdots \cap P_{n}=0$.

Now let $P$ be a minimal $\alpha$-prime ideal of $R$. Then $P_{1} P_{2} \ldots P_{n} \subseteq P$, where $P_{1}, P_{2}, \ldots, P_{n}$ are annihilator $\alpha$-prime ideals of $R$. Thus $P_{i} \subseteq P$ for some $1 \leqq i \leqq n$. Hence $P=P_{i}$ for some $1 \leqq i \leqq n$.

Conversely, suppose that $P$ is an annihilator $\alpha$-prime ideal of $R$. Let $P^{\prime}$ be an $\alpha$-prime ideal of $R$ with $P^{\prime} \subseteq P$. Suppose that rann $P \subseteq P^{\prime}$, then rann $P \subseteq P$ so that $(\operatorname{rann} P)^{2}=0$. Hence rann $P=0$. But $P$ is an annihilator $\alpha$-ideal and this is a contradiction. Therefore rann $P \not \subset P^{\prime}$. Now $P$.rann $P \subseteq P^{\prime}, P$ and rann $P$ are $\alpha$-ideals, thus $P \subseteq P^{\prime}$. Hence $P=P^{\prime}$. 
Proposition 1.7. Let $R$ be a semiprime left (or right) Goldie ring, $\alpha: R \rightarrow R$ be $a$ monomorphism. For an $\alpha$-invariant ideal $I$ of $R$, as in [14], let $I_{i}=\left\{a \in R: x^{-i} a x^{i} \in I\right\}$.

(i) Let $P$ be a minimal $\alpha$-prime ideal of $R$. Then $\Delta(P)=\bigcup_{i \geqq 0} x^{-i} P x^{i}$ is a minimal $\alpha$-prime ideal of $A(R, \alpha)$.

(ii) Let $P$ be an $\alpha$-invariant ideal of $A(R, \alpha)$. Then for each $i \geqq 0, P_{i}$ is an $\alpha$-invariant ideal of $R$. Also $P_{i}=P_{j}$ for each $j \geqq 0$.

(iii) Let $P$ be a minimal $\alpha$-prime ideal of $A(R, \alpha)$. Then $P_{i}$ is a minimal $\alpha$-prime ideal of $R$. So that there is a $1-1$ correspondence between the minimal $\alpha$-prime ideals of $R$ and of $A(R, \alpha)$ via $P \mapsto \Delta(P)$.

Proof. (i) Let $P$ be an $\alpha$-prime ideal of $R$. We show that $\Delta(P)$ is an $\alpha$-prime ideal of $A(R, \alpha)$. We have $\alpha(\Delta(P))=\Delta(P)$. Let $I$ and $J$ be $\alpha$-ideals of $A(R, \alpha)$ with $I J \subseteq \Delta(P)$ and $J \not \subset \Delta(P)$. We have $(\Delta(P))_{i}=P$, and $J_{i} \not \subset P$ for some $i \geqq 0$. Since $I_{i} J_{i} \subseteq(I J)_{i}, I_{i} J_{i} \subseteq P$, so $I_{i} \subseteq P$. We show that for each $t \geqq 0, I_{t} \subseteq P$. Let $a \in I_{t}$. Then $x^{-t} a x^{t} \in I$. If $t \geqq i$ then $x^{-i} a x^{i}=x^{-t} \alpha^{t-i}(a) x^{t}=\alpha^{t-i}\left(x^{-t} a x^{t}\right) \in I$, whence $a \in I_{i} \subseteq P$. If $t<i$, then $\alpha^{i-t}\left(x^{-i} a x^{i}\right)=$ $x^{-i} \alpha^{i-t}(a) x^{i}=x^{-t} a x^{t} \in I$, so $\alpha^{i-t}(a) \in I_{i} \subseteq P$. Thus $I \subseteq \Delta(P)$.

Now, suppose that $P$ is a minimal $\alpha$-prime ideal of $R$. By Proposition 1.6, $P=$ lann $I=\operatorname{rann} I$, for an $\alpha$-ideal $I$ of $R$. We show that $\Delta(P)=\operatorname{lann}(\Delta(I))$. To see this, let $x^{-i} a x^{i} \in \Delta(P)$, with $a \in P$. Let $x^{-j} b x^{j} \in \Delta(I)$, with $b \in I$. Then $x^{-i} a x^{i} x^{-j} b x^{j}=$ $x^{-(i+j)} \alpha^{j}(a) \alpha^{i}(b) x^{(i+j)}$, with $\alpha^{j}(a) \in P$ and $\alpha^{i}(b) \in I$. So $\Delta(P) \subseteq$ lann $\Delta(I)$. Conversely, let $x^{-i} a x^{i} \in \operatorname{lann} \Delta(I)$. Then $x^{-i} a x^{i} . \Delta(I)=0$. Let $b \in I$. Then $x^{-i} a x^{i} \cdot x^{-i} b x^{i}=0$, so $a \in$ lann $I=P$. Thus lann $\Delta(I) \subseteq \Delta(P)$. By Proposition 1.6, $\Delta(P)$ is a minimal $\alpha$-prime ideal of $A(R, \alpha)$.

(ii) The proof is straightfoward.

(iii) Let $I$ and $J$ be $\alpha$-ideals of $R$ with $I J \subseteq P_{i}$, then $\Delta(I)$ and $\Delta(J)$ are $\alpha$-ideals of $A(R, \alpha)$, with $\Delta(I) \Delta(J) \subseteq P$; so $I \subseteq P_{i}$ or $J \subseteq P_{i}$. Thus $P_{i}$ is an $\alpha$-prime ideal of $R$. By Proposition 1.6, $P=$ lann $M$ for an $\alpha$-ideal $M$ of $A(R, \alpha)$. Then $M_{i}$ is an $\alpha$-ideal of $R$. A similar argument shows that $P_{i}=\operatorname{lann} M_{i}$ and $P_{i}$ is therefore a minimal $\alpha$-prime ideal of $R$.

Corollary 1.8. $A$ ring $R$ is $\alpha$-prime if and only if $A(R, \alpha)$ is $\alpha$-prime.

\section{Semiprimitivity of $R[x ; \alpha, \delta]$}

Throughout the remainder of the paper, let $R$ be a ring with $\alpha: R \rightarrow R$ a monomorphism and $\delta: R \rightarrow R$ an $\alpha$-derivation. Let $I$ be a non-zero ideal of $R[x ; \alpha, \delta]$. For each $n \geqq 0$, let $\tau_{n}(I)=\{a \in R$ : there exists a non-zero polynomial in $I$ with degree $n$ and leading coefficient $a\} \cup\{0\}$.

Then $\tau_{n}(I)$ is a non-zero left ideal of $R$, with $\alpha\left(\tau_{n}(I)\right) \subseteq \tau_{n+1}(I)$ and $\tau_{n}(I) \supseteq \tau_{n}(I) \alpha^{n}(R)$, $n \geqq 0$.

Theorem 2.1. Let $R$ be an $\alpha$-prime left Noetherian ring. Then $R[x ; \alpha, \delta]$ is prime.

Proof. The chain $\tau_{0}(I) \subseteq \tau_{1}(I) \subseteq \cdots \subseteq \tau_{n}(I) \subseteq \ldots$, will terminate for some integer 
$p \geqq 0$. We then have $\tau_{p}(I)=\tau_{p+1}(I)$. Then $\alpha\left(\tau_{p}(I)\right) \subseteq \tau_{p}(I)$ and $\tau_{p}(I) \supseteq \tau_{p}(I) \alpha^{p}(R)$. Put $\tau_{p}(I) \cap \alpha^{p}(R)=\alpha^{p}(M)$. Then $\tau_{p}(I) \subseteq M$ and $M$ is a non-zero $\alpha$-ideal of $R$. By Lemma 1.5, rann $M$ is an $\alpha$-ideal of $R$. We have $M$. rann $M=0$, and $R$ is $\alpha$-prime, so rann $M=0$. We show that rann $I=0$. To see this, let $a_{n} x^{n}+a_{n-1} x^{n-1}+\cdots+a_{1} x+a_{0} \in$ rann $I$, with $a_{n}, a_{n-1}, \ldots, a_{1}, a_{0} \in R$. Let $b \in M$. Then $\alpha^{p}(b) \in \tau_{p}(I)$, and there exists a polynomial

$$
\alpha^{p}(b) x^{p}+b_{p-1} x^{p-1}+\cdots+b_{1} x+b_{0} \in I,
$$

with $b, b_{p-1}, \ldots, b_{1}, b_{0} \in R$, such that $\alpha^{p}(b) \alpha^{p}\left(a_{n}\right)=0$. Thus $M a_{n}=0$ and $a_{n}=0$. Therefore the result follows.

Theorem 2.2. Let $R$ be $\alpha$-prime left Noetherian with ascending chain condition on right annihilators, $\alpha: R \rightarrow R$ a monomorphism and $\delta: R \rightarrow R$ an $\alpha$-derivation. Then $R[x ; \alpha, \delta]$ is semiprimitive.

Proof. Let $J$ be the Jacobson radical of $R[x ; \alpha, \delta]$ and suppose that $J \neq 0$. As in the proof of Theorem 2.1, there exists an integer $p \geqq 0$ such that $\tau_{p}(J)$ is a non-zero left ideal of $R$ with $\alpha\left(\tau_{p}(J)\right) \subseteq \tau_{p}(J)$ and $\tau_{p}(J) \supseteq \tau_{p}(J) \alpha^{p}(R)$. Now, the subset $\Delta=\bigcup_{i \geqq p} x^{i} \tau_{p}(J) x^{i}$ of $A(R, \alpha)$ is an ideal of $A(R, \alpha)$. To see this, let $x^{-i} a x^{i} \in \Delta, x^{-j} r x^{j} \in A(R, \alpha)$, with $i \geqq P, j \geqq 0$, $a \in \tau_{p}(J)$ and $r \in R$. Then $x^{-i} a x^{i} \cdot x^{-j} r x^{j}=x^{-(i+j)} \alpha^{j}(a) \alpha^{i}(r) x^{(i+j)}$. We have $\alpha^{j}(a) \in \tau_{p}(J)$ and $\alpha^{j}(a) \alpha^{i}(r) \in \tau_{p}(J) \alpha^{i}(R) \subseteq \tau_{p}(J)$.

We show that every element of $\Delta$ is a zero divisor. Let $x^{-i} a x^{i} \in \Delta, i \geqq p$ and $a \in \tau_{p}(J)$. Then for some polynomial $f(x)=a x^{p}+a_{p-1} x^{p-1}+\cdots+a_{1} x+a_{0} \in J$ and a polynomial $g(x)=c_{q} x^{q}+c_{q-1} x^{q-1}+\cdots+c_{1} x+c_{0} \in R[x ; \alpha, \delta]$, with $c_{q} \neq 0$, we have $(1+f(x) x) g(x)=1$. By comparing the leading term in this equation we have $a \alpha^{p+1}\left(c_{q}\right)=0$, so $x^{-i} a x^{i} \cdot x^{-i} \alpha^{p+1}\left(c_{q}\right) x^{i}=0$. Thus $x^{-i} a x^{i}$ is a zero divisor. Since $R$ is left Noetherian with ascending chain condition on right annihilators, by [7], we have $\alpha(N(R)) \subseteq N(R)$. But $R$ is $\alpha$-prime, and $\{N(R)\}^{n}=0$ for some $n \geqq 0$. Then $R$ is semiprime. By [14, Corollary 7.5], $A(R, \alpha)$ is semiprime left Goldie. By [16, Proposition 2.3.5.], $\Delta$ cannot be essential as a left ideal. Hence there exists a left ideal $L$ of $A(R, \alpha)$ which is non zero and $\Delta . L \subseteq \Delta \cap L=0$. Since the intersection of minimal prime ideals of $A(R, \alpha)$ is zero, [16, Theorem 2.2.15], some minimal prime ideal of $A(R, \alpha)$ must contain $\Delta$. Since by Lemma 1.3, $A(R, \alpha)$ is $\alpha$-prime, minimal prime ideals of $A(R, \alpha)$ form a single orbit under $\alpha$ and yet, as above $\alpha(\Delta) \subseteq \Delta$. Therefore $\Delta=0$ and $J=0$.

Theorem 2.3. Let $R$ be a semiprime left Noetherian ring with ascending chain condition on right annihilators, $\alpha: R \rightarrow R$ a monomoprhism and $\delta: R \rightarrow R$ an $\alpha$-derivation. Then $R[x ; \alpha, \delta]$ is semiprimitive left Goldie.

Proof. By Proposition 1.6, $R$ contains finitely many minimal $\alpha$-prime ideals $P_{1}, P_{2}, \ldots, P_{n}$, say, with $P_{1} \cap P_{2} \cap \cdots \cap P_{n}=0$. Let $\mathscr{C}=\mathscr{C}_{R}(0)$ denote the set of regular elements of $R$. By Goldie's Theorem, [16, Theorem 2.3.6.], $R$ has a semisimple Artinian quotient ring $Q=\mathscr{C}^{-1} R$. By [12, Proposition 2.4]. we have $\alpha^{-1}(\mathscr{C})=\mathscr{C}$. We extend $\alpha$ and $\delta$ to $Q$, with

$$
\tilde{\alpha}\left(c^{-1} r\right)=\alpha(c)^{-1} \alpha(r) \text { and } \delta\left(c^{-1} r\right)=\alpha(c)^{-1} \delta(r)-\alpha(c)^{-1} \delta(r) c^{-1} r,
$$


for $c \in \mathscr{C}, r \in R$. We adapt the proof of Proposition 2.1 of Bell [3]. Here $\alpha$ is not assumed to be surjective. Let $1 \leqq i \leqq n$. By [16, Proposition 2.1.16 (vi)], $Q P_{i}$ is an ideal of $Q$. By Corollary 1 of [10, Theorem 1.4.2], $Q P_{i}=Q e_{i}$ for a central idempotent $e_{i} \in Q$. Thus $e_{i}$ is the identity of $Q e_{i}$. Let $c^{-1} a \in Q P_{i}$, with $c \in \mathscr{C}$ and $a \in P_{i}$. Then $\tilde{\alpha}\left(c^{-1} a\right)=$ $\alpha(c)^{-1} \alpha(a) \in Q P_{i}$, because $\alpha^{-1}\left(P_{i}\right)=P_{i}$ and $\alpha(c) \in \mathscr{C}$. Thus $\tilde{\alpha}\left(Q P_{i}\right) \subseteq Q P_{i}$. We have $\tilde{\alpha}\left(e_{i}\right) e_{i}=e_{i}$ and $\left(\tilde{\alpha}\left(e_{i}\right)-e_{i}\right) e_{i}=0$, so $\tilde{\alpha}\left(e_{i}\right)=e_{i}$. We have also,

$$
\tilde{\delta}\left(e_{i}\right)=\tilde{\delta}\left(e_{i}^{2}\right)=\tilde{\delta}\left(e_{i}\right) e_{i}+\tilde{\alpha}\left(e_{i}\right) \tilde{\delta}\left(e_{i}\right)=2 e_{i} \tilde{\delta}\left(e_{i}\right)
$$

Hence $e_{i} \delta\left(e_{i}\right)=2 e_{i}^{2} \tilde{\delta}\left(e_{i}\right)=2 e_{i} \delta\left(e_{i}\right)$, so $e_{i} \tilde{\delta}\left(e_{i}\right)=0$ and $\tilde{\delta}\left(e_{i}\right)=2 e_{i} \delta\left(e_{i}\right)=0$. Thus $\tilde{\delta}\left(Q P_{i}\right)=$ $\tilde{\delta}\left(\mathscr{C}^{-1} P_{i}\right)=\tilde{\delta}\left(Q e_{i}\right) \subseteq Q e_{i}=\mathscr{C}^{-1} P_{i}$ and $\delta\left(P_{i}\right) \subseteq \mathscr{C}^{-1} P_{i} \cap R=P_{i}$. Therefore for each $1 \leqq i \leqq n$, $\alpha^{-1}\left(P_{i}\right)=P_{i}$ and $\delta\left(P_{i}\right) \subseteq P_{i}$. This implies that $P_{i} R[x ; \alpha, \delta]$ is an ideal of $R[x ; \alpha, \delta]$.

There are induced monomorphisms and derivations $\bar{\alpha}_{i}$ and $\bar{\delta}_{i}$ on $R / P_{i}$, and we have

$$
R[x ; \alpha, \delta] / P_{i} R[x ; \alpha, \delta] \cong\left(R / P_{i}\right)\left[x ; \bar{\alpha}_{i}, \bar{\delta}_{i}\right]
$$

For each $1 \leqq i \leqq n, R / P_{i}$ is $\tilde{\alpha}_{i}$-prime left Noetherian. We show that the ascending chain condition on annihilator right ideals passes to $R / P_{i}$, for each $1 \leqq i \leqq n$. By Proposition 1.6, $P_{i}=\operatorname{rann}\left(K_{i}\right)$, with $K_{i}$ an $\alpha$-ideal of $R$, for $1 \leqq i \leqq n$. We have $P_{i}$ is an annihilator $\alpha$-ideal. Let $\overline{0} \neq \bar{M} \subseteq R / P_{i}$ be a right annihilator in $R / P_{i}$. We show that $M=$ $\{a \in R: \bar{a} \in \bar{M}\}$ is a right annihilator in $R$. Suppose that $\bar{M}=\operatorname{rann} \bar{I}$. Let $I$ be the inverse image of $\bar{I}$ in $R$. We have $\bar{I} \neq 0$, otherwise $\bar{M}=R / P_{i}$.

Thus $I \not P_{i}$, so $K_{i} I \neq 0$. We show that $M=\operatorname{rann}\left(K_{i} I\right)$. Since $\bar{I} \bar{M}=0, K_{i} I M=0$. Hence $I M \subseteq P_{i}$. Also, if $K_{i} I a=0$, then $I a \subseteq P_{i}$, so $\bar{I} \bar{a}=0$ and $\bar{a} \in \bar{M}$. Now, let $\bar{M}_{1} \subseteq \bar{M}_{2} \subseteq \cdots \subseteq$ $\bar{M}_{n} \subseteq \cdots$, be a chain of right annihilators in $R / P_{i}$. By above, $M_{1} \subseteq M_{2} \subseteq \cdots \subseteq M_{n} \ldots$, is a chain of right annihilators in $R$, which terminates. Therefore for each $1 \leqq i \leqq n, R / P_{i}$ is a left Noetherian ring satisfying the ascending chain condition on right annihilators. By Theorem 2.2, $\left(R / P_{i}\right)\left[x ; \bar{\alpha}_{i}, \bar{\delta}_{i}\right]$ is semiprimitive. Thus $P_{i} R[x ; \alpha, \delta]$ is a semiprimitive ideal of $R[x ; \alpha, \delta]$. Since these ideals have zero intersection, $R[x ; \alpha, \delta]$ is semiprimitive.

By Goldie's theorem [16, Theorem 2.3.6], $\mathscr{C}=\mathscr{C}_{R}(0)$ is a left Ore set in $R$ and $Q=\mathscr{C}^{-1} R$ is a semisimple Artinian ring. By [12, Proposition 2.4], $\alpha^{-1}(\mathscr{C})=\mathscr{C}$. One can show in a manner similar to that in $[9$, Lemma 1.4] that $\mathscr{C}$ is a left Ore set of regular elements in $R[x ; \alpha, \delta]$. We then have

$$
\left(\mathscr{C}^{-1} R\right)[x ; \alpha, \delta] \cong \mathscr{C}^{-1}(R[x ; \alpha, \delta])
$$

By [5, Theorem 3.2], $Q[x ; \alpha, \delta])$ is semiprime left Noetherian. We have that $Q$ is $\alpha$-prime, so by Theorem 2.3, $Q[x ; \alpha, \delta]$ is prime left Noetherian. By [4, Proposition 2.3], 0 is a left Goldie ideal of $R[x ; \alpha, \delta]$, which means that $R[x ; \alpha, \delta]$ is left Goldie.

Theorem 2.5. Let $R$ be a semiprime Goldie ring, $\alpha: R \rightarrow R$ a monomorphism such that $\alpha$ extends to an automorphism $\bar{\alpha}$ of the quotient ring $Q(R)$ of $R$. Let $\delta: R \rightarrow R$ be an $\alpha-$ derivation, then $R[x ; \alpha, \delta]$ is semiprimitive left Goldie.

Proof. By Proposition 1.6, $R$ contains finitely many minimal $\alpha$-prime ideals 
$P_{1}, P_{2}, \ldots, P_{n}$, say, with $P_{1} \cap P_{2} \cap \cdots \cap P_{n}=0$. For each $1 \leqq i \leqq n, P_{i}$ is an annihilator $\alpha$-prime ideal. By Theorem 2.3, we have

$$
R[x ; \alpha, \delta] / P_{i} R[x ; \alpha, \delta] \cong\left(R / P_{i}\right)[x ; \bar{\alpha}, \bar{\delta}] \text {, and } \bigcap_{i=0} P_{i} R[x ; \alpha, \delta]=0
$$

Let $\bar{S}=\left\{\bar{c} \in \bar{R}=R / P_{i}: c\right.$ is regular in $\left.R\right\}$. We show that $\bar{S}$ consists of regular elements of $\bar{R}=R / P_{i}$ and satisfies the left Ore condition. To see this, let $\bar{c} \bar{r}=0$, with $c \in \mathscr{C}, r \in R$. Then $c r \in P_{i}$. But $P_{i}$ is an annihilator $\alpha$-ideal, so for an ideal $A$ of $R / P_{i}$, we have $P_{i}=$ lann $A=\operatorname{rann} A$. Then $A r c=0$ implies that $r \in P_{i}$. Also, since $P_{i}=$ lann $A, \bar{r} \bar{c}=0$, gives $\bar{r}=\overline{0}$. Now, if $\bar{c} \in \bar{S}$ and $\bar{a} \in \bar{R}$, then $c$ is regular in $R$. So there is $b \in R$ and $d \in \mathscr{C}_{R}(0)$ such that $d a=b c$. Hence $\bar{d} \bar{a}=\bar{b} c$, and by [16, Theorem 2.1.12], $\bar{R}_{\bar{s}}$ exists.

Now, let $a \in R$. Let $\tilde{\alpha}$ be as in the proof of Theorem 2.3. Since $\tilde{\alpha}^{n}$ is an automorphism of $Q(R)$, there is for each $n \geqq 0, b \in R$ and $c \in \mathscr{C}_{R}(0)$ such that $a=\tilde{\alpha}^{n}\left(b c^{-1}\right)$. Thus, there exists $c \in \mathscr{C}$ such that, for each $n \geqq 0, a \alpha^{n}(c) \in \alpha(R)$. Therefore for each $\bar{a} \in \bar{R}$, and $n \geqq 0$, there exists $\bar{c} \neq 0$ such that $\bar{a} \bar{\alpha}^{n}(\bar{c})=\bar{\alpha}^{n}(\bar{b})$ with $\bar{b} \in \bar{R}$. Since $\alpha^{-1}(\mathscr{C})=\mathscr{C}, \bar{\alpha}^{n}(\bar{c})$ is regular in $R / P_{i}$. So $\bar{b} \neq \overline{0}$.

Let $\bar{J}=J(\bar{R}[x ; \bar{\alpha}, \bar{\delta}])$, with $\bar{R}=R / P_{i}$. Let $\bar{J} \neq 0$. By the above argument

$$
\tau=\left\{a \in \bar{R}: \bar{\alpha}^{n}(a) x^{n}+a_{n-1} x^{n-1}+\cdots+a_{1} x+a_{0} \in \bar{J}, \text { for some } n>0\right\}
$$

is non-zero. Also $\tau$ is an $\bar{\alpha}$-ideal of $\bar{R}$. Then $\Delta(\tau)=\bigcup_{i \geqq 0} x^{-i} \tau x^{i}$ is an $\bar{\alpha}$-ideal of $A(\bar{R}, \bar{\alpha})$.

Now, one can show that

$$
A\left(R / P_{i}, \bar{\alpha}\right) \cong A(R, \alpha) / \Delta\left(P_{i}\right)
$$

with $a^{-i} \bar{a} x^{i} \mapsto x^{-i} a x^{i}+\Delta\left(P_{i}\right)$, where $\Delta\left(P_{i}\right)=\bigcup_{i \geqq 0} x^{-i} P_{i} x^{i}$.

By [14, Corollary 7.5], $A(R, \alpha)$ is semiprime left Goldie. By Proposition 1.7, $\Delta\left(P_{i}\right)$ is a minimal $\alpha$-prime ideal of $A(R, \alpha)$. So $\Delta\left(P_{i}\right)$ is a finite intersection of minimal prime ideals of $A(R, \alpha)$. Using [16, Proposition 3.2.5], one can show that $A(R, \alpha) / \Delta\left(P_{i}\right)$ is $\alpha$-prime left Goldie. So $A\left(R / P_{i}, \alpha\right)$ is semiprime left Goldie. Hence $\Delta(\tau)$ cannot be essential as a left ideal of $A\left(R / P_{i}, \bar{\alpha}\right)$. Therefore as in the proof of Theorem 2.3, the result follows.

\section{Semiprimitivity of $R \mid x ; \alpha]$}

Let $R$ be a ring, $\alpha: R \rightarrow R$ a monomorphism. Let $f(x)=\sum_{i=m}^{n} a_{i} x^{i} \in R[x ; \alpha]$, with $a_{n} \neq 0$ and $a_{m} \neq 0, n \geqq 0, m \geqq 0$. The length of $f(x)$ is the non-negative integer $n-m$. For an ideal $I$ of $R[x ; \alpha]$ we denote by $\mu(I)$ the set of non-zero elements of $I$ of minimal length. We note that the set $\mu(I) \cup\{0\}$ is closed under multiplication on either side by elements of $R[x ; \alpha]$ of length 0 , i.e. elements of the form $r x^{i}, r \in R, i \geqq 0$.

The following lemma is proved in [13], where $\alpha$ is assumed to be an automorphism. However the proof in [13] remains valid in our more general situation.

Lemma 3.2. Let $J$ be the Jacobson radical of $R[x ; \alpha]$. Let $f(x)=\sum_{i=m}^{n} a_{i} x^{i} \in \mu(J)$ be such that $m>0$. Then there exists an integer $s>0$ such that $a_{n} \alpha^{n}\left(a_{n}\right) \alpha^{2 n}\left(a_{n}\right) \ldots \alpha^{s n}\left(a_{n}\right)=0$. 
Definition 3.3. Let $R$ be a ring, $\alpha: R \rightarrow R$ a monomorphism. Then the element $r \in R$ is said to be $\alpha$-nilpotent if for all integers $k>0$, there exists a positive integer $s=s(r, k)$ such that

$$
r \alpha^{k}(r) \alpha^{2 k}(r) \ldots \alpha^{s k}(r)=0
$$

An ideal (left ideal) of $R$ is said to be $\alpha$-nil, if every element of $I$ is $\alpha$-nilpotent.

Theorem 3.4. Let $R$ be a semiprime right Goldie ring. Let $\alpha: R \rightarrow R$ be a monomorphism. Then $R[x ; \alpha]$ is semiprimitive.

Proof. Let $J$ be the Jacobson radical of $R[x ; \alpha]$ and assume that $J \neq 0$. Let $\tau=\{a \in R$ : there exists a polynomial of minimal length in $J$ with $a$ its leading coefficient $\} \cup\{0\}$, which is non-zero left ideal of $R$, with $\alpha(\tau) \subseteq \tau$. By Lemma 3.2, $\tau$ is $\alpha$ nil. By [14, Corollary 7.5], $A(R, \alpha)$ is semiprime right Goldie. Let $\Delta=\bigcup_{i \geq 0} x^{-i} \tau x^{i}$. Then $\Delta$ is an $\alpha$-nil left ideal of $A(R, \alpha)$. Suppose that $\Delta$ is not a nil left ideal of $A(R, \alpha)$. We adapt the proof of Theorem 2.1 of Ram [18], to show that for any $a \in \Delta$ which is a nonnilpotent element, there exists a positive integer $n$ such that $a \alpha^{n}(a) \neq 0$. To see this let us suppose that $a \alpha^{n}(a)=0$ for every positive integer $n$. Define

$$
I_{m}=a A(R, \alpha)+\alpha^{-1}(a) A(R, \alpha)+\cdots+\alpha^{-m}(a) A(R, \alpha)
$$

Note that $\alpha$ is an automorphism of $A(R, \alpha)$. Then we have

$$
I_{0} \subseteq I_{1} \subseteq \cdots \subseteq I_{m} \cdots,
$$

and

$$
\text { lann } I_{0} \supseteq \operatorname{lann} I_{1} \supseteq \cdots \supseteq \operatorname{lann} I_{m} \supseteq \cdots
$$

But the ascending chain condition on right annihilators is equivalent to the descending chain condition on left annihilators. Therefore for some positive integer $t$, lann $I_{t}=$ lann $I_{t+1}$. Now, $a \alpha^{n}(a)=0$ for each $n \geqq 1$ if and only if $\alpha^{-n}(a) a=0$ for each $n \geqq 1$. Since $\alpha^{-(t+1)}$ (a) Elann $I_{t}, \alpha^{-(t+1)}(a) \in \operatorname{lann} I_{t+1}$. Thus $\alpha^{-(t+1)}(a) \alpha^{-(t+1)}(a)=0$ and $a^{2}=0$. This contradicts the assumption that $a$ is not nilpotent. Thus, there exists $n \geqq 1$ such that $a \alpha^{n}(a) \neq 0$.

Since $\Delta$ is $\alpha$-nil, for some positive integer $s \geqq 1, a \alpha^{n}(a) \ldots \alpha^{s n}(a)=0$. Let $s$ be the least such integer. We have $\alpha^{-s n}(a) \alpha^{(1-s) n}(a) \ldots \alpha^{-n}(a) a=0$. Put $u=\alpha^{(1-s) n}(a) \ldots \alpha^{-n}(a) a$. Then $u \neq 0$. If the left ideal $A(R, \alpha) u$ is not nil, then $b u$ is not nilpotent for some $b \in A(R, \alpha)$. Put $a_{1}=a$ and $a_{2}=b u$. We show that $\operatorname{rann}\left(a_{1}\right) \varsubsetneqq \operatorname{rann}\left(a_{2}\right)$. Since $\alpha^{-s n}(a) \alpha^{(1-s) n}$ (a) $\ldots \alpha^{-n}(a) a=0, \alpha^{(1-s) n}(a) \ldots \alpha^{-n}(a) a \alpha^{n}(a)=0$. Hence $a_{2} \alpha_{n}(a)=0$. But $a \alpha^{n}(a) \neq 0$, thus $\operatorname{rann}\left(a_{1}\right) \varsubsetneqq \operatorname{rann}\left(a_{2}\right)$. Replace $a=a_{1}$ by $a_{2}$ and $a_{2} \alpha^{n_{2}}\left(a_{2}\right) \neq 0$ for some $n_{2}$. Repeat the argument to get $a_{3}$ such that $\operatorname{rann}\left(a_{1}\right) \varsubsetneqq \operatorname{rann}\left(a_{2}\right) \varsubsetneqq \operatorname{rann}\left(a_{3}\right)$. Continuing in this way we 
get a strictly ascending chain of right annihilators in $R$. This is a contradiction. Therefore there is a non-zero nil left ideal in $A(R, \alpha)$. Since $A(R, \alpha)$ is semiprime right Goldie, then by $[15$, Theorem 1] every nil left ideal is nilpotent. Therefore $R[x ; \alpha]$ is semiprimitive.

Remark. Since $\alpha$ is not assumed to be surjective on $R$, the assymmetry of the construction of $R[x ; \alpha]$ means that symmetry cannot be cited to give the next result as a corollary of Theorem 3.4 .

Theorem 3.5. Let $R$ be a semiprime left Goldie ring and $\alpha: R \rightarrow R$ a monomorphism of $R$. Then $R[x ; \alpha]$ is semiprimitive.

Proof. Since $R$ is semiprime left Goldie, so it has a semisimple Artinian quotient ring, by [16, Theorem 2.3.6]. Hence one can show that $R$ satisfies the ascending chain condition on right annihilators. Also $A(R, \alpha)$ is semiprime left Goldie. The rest of the proof is similar to the proof of Theorem 3.4 .

Corollary 3.6. Let $R$ be a left Noetherian ring satisfying the ascending chain condition on right annihilators, $\alpha: R \rightarrow R$ a monomorphism, then

$$
J(R[x ; \alpha])=N(R[x ; \alpha])=N(R)[x ; \alpha]
$$

Proof. By [7] we have $\alpha(N(R)) \subseteq N(R)$. So $N(R)[x ; \alpha]$ is an ideal of $R[x ; \alpha]$. We show that $\alpha^{-1}(N(R))=N(R)$. To see this let $\alpha(a) \in N(R), a \in R$. Then $(R \alpha(a) R)^{n}=0$ for some $n \geqq 0$. It follows that $R a R$ is a nil ideal of $R$, so we have $\alpha^{-1}(N)=N$. Let $\bar{\alpha}$ be the homomorphism induced on $R / N(R)$, by $\alpha$, given by $\bar{\alpha}(a+N(R))=\alpha(a)+N(R)$. Then $\bar{\alpha}$ is injective. We have $R / N(R)$ is semiprime left Noetherian and $\bar{\alpha}$ is a monomorphism of $R / N(R)$. By Theorem $3.4,(R / N(R))[x ; \bar{\alpha}]$ is semiprimitive. We have

$$
(R / N(R))[x ; \bar{\alpha}] \cong R[x ; \alpha] / N(R)[x ; \alpha]
$$

Thus $N(R)[x ; \alpha]$ is a semiprimitive ideal of $R[x ; \alpha]$ and $J(R[x ; \alpha]) \subseteq N(R)[x ; \alpha]$.

Since $\alpha(N(R)) \subseteq N(R)$ and $\{N(R)\}^{k}=0$ for some $k>0,(N(R)[x ; \alpha])^{k}=0$. So the result follows.

Corollary 3.7. Let $R$ be a right Noetherian ring satisfying the ascending chain condition on left annihilators. Then we have the same result as Corollary 3.6.

Example 3.8. There are examples which show that some conditions on $R$ and $\alpha$ are necessary if results of the nature of Theorems $2.2,2.3$ and 3.4 are to be valid. One is the example constructed by Jordan $[13, \S 5]$. In that example 0 is a semiprime ideal of $R$ which is $\alpha$-prime but it is not strongly $\alpha$-prime. But $R[x ; \alpha]$ has a non zero nil ideal. Another example is from Pearson and Stephenson in $[17, \S 2]$. They have constructed an $\alpha$-prime commutative ring, and automorphism $\alpha$ of $R$ such that $R[x ; \alpha]$ is prime with a non zero nil ideal. 
Acknowledgements. I am grateful to my supervisor Dr D. A. Jordan for his help and constant encouragement during the preparation of this paper. Thanks are also due to the referee for his comments. In addition thanks are due to the Ministry of Higher Education of Iran for financial support.

\section{REFERENCES}

1. S. A. Amitsur, Radical of polynomial rings, Canad. J. Math. 8 (1956), 355-361.

2. S. S. BEDI and J. RAM, Jacobson radical of skew polynomial rings and group rings, Israel $J$. Math. 35 (1980), 327-338.

3. A. D. Bell, When are all prime ideals in an Ore extension Goldie?, Comm. Algebra 13 (1985), 1743-1762.

4. A. D. BeLl, Goldie dimensions of prime factors of polynomial and skew polynomial rings, $J$. London Math. Soc. (2) 29 (1984), 418-424.

5. G. Cauchon and J. C. Robson, Endomorphisms, derivations and polynomial rings, $J$. Algebra 53 (1978), 227-238.

6. A. W. Chatters and C. R. Hajarnavis, Rings with chain conditions (Research Notes in Mathematics 44, Pitman, London, 1980).

7. C. Dean, Monomorphisms and Radicals of Noetherian rings, J. Algebra 99, (1986), 573-576.

8. A. El Ahmar, Anneaux des polynomes de Ore sur des anneaux de Jacobson, Rev. Roumaine Math. Pures Appl. 26 (1981), 1277-1286.

9. A. W. Goldie and G. Michler, Ore extensions and polycyclic group rings, J. London Math. Soc. (2) 9 (1974), 337-345.

10. I. N. Herstein, Topics in Ring theory (The University of Chicago Press, 1969).

11. R. S. Irving, Prime ideals of Ore extensions over commutative rings, J. Algebra 56 (1979), 315-342.

12. A. V. Jategaonkar, Skew polynomial rings over orders in Artinian rings, J. Algebra 21 (1972), 51-59.

13. C. R. Jordan and D. A. Jordan, A note on semiprimitivity of Ore extensions, Comm. Algebra 4 (1976), 647-656.

14. D. A. Jordan, Bijective extensions of injective ring endomorphisms, J. London Math. Soc. (2) 25 (1982), 435-448.

15. C. Lanskı, Nil subrings of Goldie rings are nilpotent, Canad. J. Math. 21 (1969), 904-907.

16. J. C. MCConnell and J. C. Robson, Non-commutative Noetherian rings (John Wiley \& Sons, Chichester, 1987).

17. K. R. Pearson and W. Stephenson, A skew polynomial ring over a Jacobson ring need not be a Jacobson ring, Comm. Algebra 5 (1977), 783-794.

18. J. Ram, On the semiprimitivity of skew polynomial rings, Proc. Amer. Math. Soc. (3) 90 (1984), 347-351.

Department of Pure Mathematics

UNIVERSITY OF SHEFFIELD

SHEFFIELD S3 7RH
Department of Mathematics TARBIAT ModarRes UnIVERSITY P.O. Box 14155-4838

TeHran IRAN 